Relations industrielles

Industrial Relations

\title{
Industrial and Labor Relations review
}

\section{Gaston Cholette}

Volume 5, numéro 1, septembre-octobre 1949

URI : https://id.erudit.org/iderudit/1023268ar

DOI : https://doi.org/10.7202/1023268ar

Aller au sommaire du numéro

Éditeur(s)

Département des relations industrielles de l'Université Laval

ISSN

0034-379X (imprimé)

1703-8138 (numérique)

Découvrir la revue

Citer cet article

Cholette, G. (1949). Industrial and Labor Relations review. Relations

industrielles / Industrial Relations, 5(1), 10-10. https://doi.org/10.7202/1023268ar

Tous droits réservés (C Département des relations industrielles de l’Université Laval, 1949
Ce document est protégé par la loi sur le droit d'auteur. L'utilisation des services d'Érudit (y compris la reproduction) est assujettie à sa politique d'utilisation que vous pouvez consulter en ligne.

https://apropos.erudit.org/fr/usagers/politique-dutilisation/ 


\section{Les reunes en relations industrielles}

Dans le but de rendre de plus grands services d ses lecteurs et de rencontrer davantage les exigences dune publication universitaire, le Bulletin des relations industrielles entreprend, dans ce numéro, de présenter les principales revues dexpression française et anglaise sur les relations du travail.

La méthode provisoirement adoptée, pour le début, consiste d attirer l'attention sur deux ou trois revues d la fols, en faisant, grosso modo, une recension des numéros de l'année précédente. Il ne s'agit pas, comme on le voit, d'une chronique de revues proprement dite, dans laquelle on s'attacherait plutốt d signaler et d̀ analyser les principales études parues récemment.

La Rédaction du Bulletin espère que cette initiative saura plaire et que, malgré limperfection inévitable de la formule actuelle, les lecteurs $y$ trouveront leur profit.

\section{INDUSTRIAL AND LABOR RELATIONS REVIEW}

«Industrial and Labor Relations Review » est une publication trimestrielle d'environ 150 pages, préparée par la New York State School of Industrial and Labor Relations, Cornell University, Ithaca, New York.

Bien qu'elle n'ait que deux années d'existence, c'est sûrement l'une des revues américaines les plus réputées dans le domaine des relations du travail, dont elle aborde à peu près tous les aspects. Chaque numéro contient quatre ou cinq articles de fond, une section désignée sous le titre de "discussions et communications", une partie documentaire, une liste des publications récentes très élaborée et bien divisée en catégories spéciales, une chronique de livres, des nouvelles et des notes.

La présente chronique porte sur les quatre numéros du volume 2, soit ceux d'octobre 1948, de janvier, d'avril et de juillet 1949. Dans certains de ces numéros, plusieurs des articles portent sur un thème commun.

Celui d'octobre 1948 contient plusieurs études intéressantes sur les réformes de structure dans l'entreprise. Voici les titres des principaux articles de cette livraison: - Union Management Co-operation in the Millinery Manufacturing in the New York Area », \& Profit Sharing under Collective Bargaining », «conomic and Legal Projects of Profit Sharing Plans », « The Small Business Enterprise and the Management Prerogative Issue ».

Ce dernier article a été rédigé par J.G. Turnbull, un membre du « Social Science Research Council», à la suite d'une enquête faite auprès de vingt-quatre entreprises. L'auteur énumère et classifie les diverses séries d'opinions et d'attitudes des patrons concernant la tendance des syndicats ouvriers à une intégration de plus en plus intime dans l'entreprise par la participation à la propriété, à la gestion et aux bénéfices.

L'auteur ne porte pas lui-même de jugement de valeur sur ces questions - du moins il s'efforce d'agir ainsi mais il expose le plus objectivement possible le résultat de ses entretiens avec les représentants patronaux.

En conclusion de son article, l'auteur fait quelques suggestions qui lui semblent opportunes sur les formes de collaboration patronale-ouvrière. Cette étude projette un reflet fidèle de la mentalité des chefs d'industries américaines sur le problème de la propriété et de l'autorité dans l'entreprise.

Deux des articles du numéro de janvier 1949 traitent de la productivité. L'un d'entre eux est intitulé: -Union-Management Co-operation and Productivity.

Dans la livraison d'avril 1949, trois des quatre articles portent sur des problèmes courants de la législation du travail.
Enfin, le numéro de juillet 1949 contient des études sur la fonction économique des grèves, l'atelier fermé et la direction du personnel.

Le prix de l'abonnement à cette revue est de $\$ 4.00$ par année.

G. C.

\section{BULLETIN SOCIAL DES INDUSTRIELS}

Le \& Bulletin social des industriels 》 est une revue mensuelle d'une quarantaine de pages publiée par l'Association des patrons et ingénieurs catholiques de Belgique.

Comme l'association dont elle est l'organe officiel, cette publication a pour but primordial de faire prendre conscience aux patrons catholiques de leurs responsabilités et de leur fonction sociales, en plus de chercher des formules d'application de la doctrine sociale de l'Eglise. Ce n'est pas un moyen de défense mais un instrument de formation.

On y trouve fréquemment des études philosophiques très poussées et fort originales sur des sujets comme les suivants: «Le salaire familial et la pensée des encycliques 》, «L'autorité dans l'entreprise », «Economie de marché ou économie de commandement», «Que faut-il entendre par entreprise? », « Les relations industrielles et la psychologie sociale », etc.

A côté de ces travaux théoriques, il y a souvent des articles qui décrivent et commentent certaines expériences patronales-ouvrières intéressantes, par exemple: «Quelques expériences de dépassement de salariat », «Une expérience de participation aux économies $\gg$, etc.

Les collaborateurs du « Bulletin social des industriels 》 sont habituellement des universitaires et des chefs d'entreprises réputées, ce qui lui donne une grande valeur théorique et pratique.

Chaque numéro contient un éditorial, deux ou trois articles de fond et des chroniques diverses. On y trouve presque régulièrement une rubrique consacrée à l'actualité et à la documentation. Dans la plupart des numéros il y a aussi des échos de journées d'études patronales.

Le «ulletin social des industriels 》 s'adresse tout spécialement aux patrons catholiques, mais les sujets dont il traite et la façon dont il les aborde dans le contexte d'un pays où les expériences d'application de la doctrine sociale de l'Eglise comptent parmi les plus généreuses et les plus originales, sont de nature à intéresser grandement tous ceux qui cherchent ardemment et sincèrement des exemples ou des formules de réformes de structure sur le plan de l'entreprise, de la profession et de la nation.

On s'abonne au \& Bulletin social des industriels 》 en versant la somme de 300 francs au Secrétariat général de l'Association des patrons et ingénieurs catholiques de Belgique, 17, rue du Gouvernement provisoire, Bruxelles. 